\title{
Tau in biofluids - relation to pathology, imaging and clinical features
}

Henrik Zetterberg, MD, $\mathrm{PhD}^{1,2,3}$

${ }^{1}$ Clinical Neurochemistry Laboratory, Sahlgrenska University Hospital, Mölndal, Sweden

${ }^{2}$ Department of Psychiatry and Neurochemistry, Institute of Neuroscience and Physiology, the Sahlgrenska Academy at the University of Gothenburg, Mölndal, Sweden

${ }^{3}$ Department of Molecular Neuroscience, UCL Institute of Neurology, Queen Square, London, UK

Address for correspondence:

Henrik Zetterberg, MD, PhD

Department of Psychiatry and Neurochemistry

The Sahlgrenska Academy at the University of Gothenburg

S-431 80 Mölndal

SWEDEN

Tel (office): +46313430142

Tel (cell): +46768672647

Tel (secretary): +46313430025

Fax: +46 31419289

E-mail: henrik.zetterberg@gu.se

Key words: tau, cerebrospinal fluid, plasma, biomarker, Alzheimer's disease 


\begin{abstract}
Tau is a microtubule-binding protein that is important for the stability of neuronal axons. It is normally expressed within neurons and is also secreted into the brain interstitial fluid that communicates freely with cerebrospinal fluid (CSF) and, in a more restricted manner, blood via the glymphatic clearance system of the brain. In Alzheimer's disease (AD), neuroaxonal degeneration results in increased release of tau from neurons. Further, tau is truncated and phosphorylated, which leads to aggregation of tau in neurofibrillary tangles of the proximal axoplasm. Neuroaxonal degeneration and tangle formation are reflected by increased concentrations of total tau (T-tau, measured using assays that detect most forms of tau) and phospho-tau (P-tau, measured using assays with antibodies specific to phosphorylated forms of tau). In AD CSF, both T-tau and P-tau concentrations are increased. In stroke and other CNS disorders with neuroaxonal injury but without tangles, T-tau is selectively increased, whilst P-tau concentration often stays normal. In tauopathies (diseases with both neurodegeneration and neurofibrillary tangles) other than AD, CSF T-tau and P-tau concentrations are typically unaltered, which is a puzzling result that warrants further investigation. In the current review, I discuss the association of T-tau and P-tau concentrations in body fluids with neuropathological changes, imaging findings and clinical features in AD and other CNS diseases.
\end{abstract}




\section{Background}

In 1986, one year after the sequencing of amyloid $\beta(\mathrm{A} \beta)$ in senile plaques in Alzheimer's disease $(\mathrm{AD})$ brains [1], data were published showing that neurofibrillary tangles (the other major neuropathological hallmark of AD) are composed of abnormally phosphorylated and truncated forms of tau $[2,3]$. Tau is a microtubule-binding axonal protein that promotes microtubule assembly and stability [4]. Abnormal phosphorylation and truncation of tau may lead to disassembly of microtubules and impaired axonal transport with compromised neuronal function and tau aggregation into paired helical filaments and neurofibrillary tangles [5]. Apart from executing important intracellular functions, tau is normally secreted from neurons into the brain interstitial fluid $[6,7]$. This fluid communicates freely with cerebrospinal fluid (CSF) and, in a more restricted manner that is regulated by the glymphatic clearance system of the brain, with blood, where tau may be enzymatically degraded and cleared from the body by unknown mechanisms. Several methods to measure total tau (T-tau) and phospho-tau (P-tau) in CSF have been developed. They are reviewed in detail below and their utility in differential diagnostics of neurodegenerative diseases is summarized in Table 1.

\section{CSF T-tau}

The first CSF T-tau assay was published in 1993 [8]. This assay was a sandwich ELISA in which a monoclonal antibody against the mid-domain of tau was combined with a polyclonal anti-tau antiserum. Two years later, the first assay based on three mid-region monoclonal antibodies that recognise all tau isoforms irrespective of phosphorylation state was published [9]. AD patients displayed clearly increased T-tau concentrations in their CSF [8,9], a finding that has been replicated in hundreds of papers, using different assays, in many different clinical contexts [10]. In response to acute brain injury, CSF T-tau concentrations are dynamic; they increase during the first few days following the injury and stay elevated for some weeks until they normalise $[11,12]$. This has led to the view that increased CSF T-tau concentration reflects ongoing axonal injury or degeneration, which in turn may indicate disease intensity. In AD-type neurodegeneration, CSF T-tau concentrations are stably increased over many years [13].

\section{CSF P-tau}

The first CSF assay for P-tau, the form of tau that is thought to represent neurofibrillary tangles, was published in 1995 [9]. Since then, P-tau assays for different forms of phosphorylated tau protein have been examined [14]. They correlate well and associate with 
$\mathrm{AD}$ in a similar manner [14]. There are at present only 3 conditions in addition to $\mathrm{AD}$ in which elevated CSF P-tau levels have been reported, none of which is an important differential diagnosis of $\mathrm{AD}$ : (i) in term and pre-term newborns, possibly reflecting physiological tau phosphorylation in brain development [15], (ii) in herpes encephalitis [16], and (iii) in superficial CNS siderosis [17, 18].

\section{CSF tau - relation to pathology}

In $\mathrm{AD}$ and normal pressure hydrocephalus, CSF P-tau concentration correlates with neurofibrillary tangle pathology $[19,20]$. The major outstanding research question regarding P-tau is why other tauopathies, like frontotemporal dementias and progressive supranuclear palsy, do not show increased CSF P-tau concentration, at least not as systematically as AD does. It is possible that these disorders show disease-specific tau phosphorylation, or that tau is processed or truncated in a way that is not recognised by currently available assays, which is an area in need of further research.

\section{CSF tau - relation to imaging}

One limitation with CSF T-tau and P-tau is that lumbar CSF concentrations will never give information on the anatomic location of the pathology. Nevertheless, it has been shown that CSF T-tau concentration correlates with structural imaging measures of hippocampal atrophy [21] and grey matter degeneration [22], which is logical given the high expression of tau in thin unmyelinated axons of the cortex [23]. Additionally, rapid progress is being made on new positron emission tomography (PET) tracers for studying tau pathology in living humans. The results so far show that CSF and PET tau markers correlate [4]. In classical AD, the two may be more or less interchangeable, but in atypical forms of AD, tau PET may be superior. This was recently illustrated in a case report that described a young patient with progressive short term memory impairment and florid sensory symptoms [24]. CSF A $\beta 42$ was abnormal and 18F-flutemetamol PET showed a distribution of amyloid- $\beta$ fibrils similar to sporadic AD. CSF T-tau and P-tau concentrations were normal, but the pattern of tau pathology, revealed using 18F-AV1451 PET, showed higher uptake in posterior cingulate, precuneus, parietal and occipital cortices, as compared to late-onset sporadic AD. Further, tau pathology exhibited a very clear inverse relationship with $18 \mathrm{~F}$-fluorodeoxyglucose metabolism, indicating neuronal hypometabolism in regions affected by tau aggregates. Genetic testing revealed a mutation of the presenilin-1 gene (Thr116Asn), previously described in a Danish family with familial AD 
[25]. Tau PET is currently being examined in regards to its usefulness to detect tauopathies other than $\mathrm{AD}$, where CSF tau markers currently fail.

In clinical trials of disease-modifying drug candidates against AD, CSF and PET tau markers may provide complementary information. Tau PET may be used to detect changes over months to years in tau pathology, whilst CSF T-tau and P-tau may be used to monitor changes in the neuronal release pattern of these molecules over weeks to months. This reasoning is presently hypothetical and waits formal testing in clinical trials.

\section{CSF tau - relation to clinical features}

CSF T-tau and P-tau are markers of disease intensity in AD; the higher concentrations, the more rapid clinical disease progression [26, 27]. The most extreme neurodegenerative condition we are aware of, Creutzfeldt-Jakob disease, is associated with very pronounced CSF T-tau elevations that are often orders of magnitude higher than what is typically seen in AD [28].

\section{Plasma tau}

Standard ELISAs for T-tau were recently transferred onto the Single molecule array (Simoa) platform, which allows for the ultrasensitive measurement of tau in blood [29]. There is so far no assay for P-tau in plasma. Plasma T-tau concentrations correlate poorly with CSF [30], but in acute hypoxic brain injury, a biphasic release of tau into the bloodstream was observed with a first peak occurring during the first few hours post-injury and a second broad peak occurring after a few more days; these increases were predictive of outcome [29]. In the dementia stage of $\mathrm{AD}$, plasma tau concentrations are slightly increased compared with cognitively normal control individuals, but not as clearly as in CSF [30], which is a wellreplicated finding [10]. In a study on concussed professional ice hockey players, plasma T-tau concentration at 1 hour post-injury was increased compared with preseason concentrations and predicted return-to-play time with high accuracy [31]. In another study of 34 patients with varying degree of traumatic brain injury (TBI), plasma tau concentrations in samples collected within 24 hours post-injury showed high accuracy to differentiate complicated mild TBI from controls (area under the curve 0.901) [32]. Recently, Olivera et al. reported increased mean plasma tau concentration in military personnel with a history of self-reported TBI compared with controls without such a history [33]. Additionally, post-concussive symptoms correlated with plasma tau concentration in the TBI group, suggesting that the increase, although 
variable, might be of pathophysiological relevance [33]. The expression of tau is brainenriched, but tau is also detectable at both mRNA and protein level in salivary glands and kidney (http://www.proteinatlas.org/ENSG00000186868-MAPT/tissue). This is an important potential confounder that may help explain the poor correlation of plasma with CSF tau. The half-life of tau also appears to be much shorter (hours) in plasma [29] than in CSF (weeks) [11].

\section{Novel fluid biomarkers for tau}

Tau proteins constitute a family of six isoforms ranging from 352-441 amino acids in size. The longest isoform in the CNS has four repeats (R1, R2, R3 and R4) and two inserts (441 amino acids in total), whereas the shortest isoform has three repeats (R1, R3 and R4) and no insert (352 amino acids in total) [34]. All of the six tau isoforms are present in an often hyperphosphorylated state in paired helical filaments from AD. In addition, there are endogenous fragments of tau; these fragments often lack different parts of the C-terminus [35]. A few years ago, ultrasensitive assays specific for 4R-tau and 3R-tau were developed [36]. Selective decreases of 4R-tau in CSF of progressive nuclear palsy and AD patients compared with controls, and lower 4R-tau levels in AD compared with Parkinson's disease dementia were reported, which was a promising result, but the assay has been difficult to standardize and the results are in need of replication. Recently, Lewczuk and colleagues reported increased CSF concentration of non-phosphorylated tau using a novel tau assay that specifically measures tau not phosphorylated at amino acids 175, 181 or 231 [37]. Using a new mass spectrometry workflow to enhance the detection of phosphoproteome components of $\mathrm{AD}$ brain tissue in CSF and enable the quantification of these analytes, over $75 \%$ coverage of full-length (2N4R) tau was detected in the CSF with 47 phosphopeptides covering 31 different phosphorylation sites. In a pilot study of a few AD and control CSF samples, 11 tau phosphopeptides were up-regulated by at least $40 \%$, along with an overall increase in tau levels in the CSF of AD patients relative to controls; a few phosphopeptides were downregulated [38]. Using another mass spectrometry approach, tau peptides along the entire molecule were detected in CSF and the peptide abundance pattern was similar in progressive supranuclear palsy and dementia with Lewy bodies but distinct in AD [39_ENREF_39]. These data emphasise the complexity of tau as a biomarker and warrant further investigation. 
There is as yet no certified reference method or material for the most established AD biomarkers T-tau and P-tau, but such work is currently ongoing in the International Federation of Clinical Chemistry and Laboratory Medicine working group on CSF proteins. A candidate reference method for CSF T-tau has been published [40].

\section{Conclusions}

CSF T-tau and P-tau are markers of AD-type neurodegeneration and tangle formation. Other tauopathies show mostly normal CSF tau protein concentrations, potentially due to release of other tau forms that the current assays fail to detect. One hypothesis regarding the reason for the AD-specific increase in CSF T-tau and P-tau concentrations is that neuronal CSF tau protein secretion may be induced by alzheimerogenic factors such as $A \beta 42$. Tau is a complicated biomolecule with several isoforms and fragments. More tau markers are to be expected in the near future in the light of new mass spectrometric findings. CSF and PET tau markers seem to provide complementary information on AD pathology and should as much as possible be analysed in parallel on patients in different stages of the disease, as well as in atypical forms of $\mathrm{AD}$ and in other tauopathies, to increase our knowledge on how to best interpret the two measures.

\section{Acknowledgements}

Research in the author's laboratories is funded by the Swedish Research Council, Swedish State Support for Clinical Research, the European Research Council, the Wolfson Foundation and Frimurarestiftelsen. HZ is a Wallenberg Academy Fellow.

\section{References}

1. Masters CL, Simms G, Weinman NA, Multhaup G, McDonald BL, Beyreuther K: Amyloid plaque core protein in Alzheimer disease and Down syndrome. Proc Natl Acad Sci U S A 1985, 82(12):4245-4249.

2. Grundke-Iqbal I, Iqbal K, Tung YC, Quinlan M, Wisniewski HM, Binder LI: Abnormal phosphorylation of the microtubule-associated protein tau (tau) in Alzheimer cytoskeletal pathology. Proc Natl Acad Sci U S A 1986, 83(13):4913-4917.

3. Nukina $\mathrm{N}$, Ihara $\mathrm{Y}$ : One of the antigenic determinants of paired helical filaments is related to tau protein. $J$ Biochem 1986, 99(5):1541-1544.

4. Holtzman DM, Carrillo MC, Hendrix JA, Bain LJ, Catafau AM, Gault LM, Goedert M, Mandelkow E, Mandelkow EM, Miller DS et al: Tau: From research to clinical development. Alzheimers Dement 2016.

5. Mandelkow EM, Mandelkow E: Biochemistry and cell biology of tau protein in neurofibrillary degeneration. Cold Spring Harb Perspect Med 2012, 2(7):a006247. 
6. Saman S, Kim W, Raya M, Visnick Y, Miro S, Jackson B, McKee AC, Alvarez VE, Lee NC, Hall GF: Exosome-associated tau is secreted in tauopathy models and is selectively phosphorylated in cerebrospinal fluid in early Alzheimer disease. $J$ Biol Chem 2012, 287(6):3842-3849.

7. Maia LF, Kaeser SA, Reichwald J, Hruscha M, Martus P, Staufenbiel M, Jucker M: Changes in Amyloid-beta and Tau in the Cerebrospinal Fluid of Transgenic Mice Overexpressing Amyloid Precursor Protein. Sci Transl Med 2013, 5(194):194re 192.

8. Vandermeeren M, Mercken M, Vanmechelen E, Six J, van de Voorde A, Martin JJ, Cras P: Detection of tau proteins in normal and Alzheimer's disease cerebrospinal fluid with a sensitive sandwich enzyme-linked immunosorbent assay. J Neurochem 1993, 61(5):1828-1834.

9. Blennow K, Wallin A, Agren H, Spenger C, Siegfried J, Vanmechelen E: Tau protein in cerebrospinal fluid: a biochemical marker for axonal degeneration in Alzheimer disease? Mol Chem Neuropathol 1995, 26(3):231-245.

10. Olsson B, Lautner R, Andreasson U, Ohrfelt A, Portelius E, Bjerke M, Holtta M, Rosen C, Olsson C, Strobel G et al: CSF and blood biomarkers for the diagnosis of Alzheimer's disease: a systematic review and meta-analysis. Lancet Neurol 2016, 15(7):673-684.

11. Hesse C, Rosengren L, Andreasen N, Davidsson P, Vanderstichele H, Vanmechelen E, Blennow K: Transient increase in total tau but not phospho-tau in human cerebrospinal fluid after acute stroke. Neurosci Lett 2001, 297(3):187-190.

12. Zetterberg H, Hietala MA, Jonsson M, Andreasen N, Styrud E, Karlsson I, Edman A, Popa C, Rasulzada A, Wahlund LO et al: Neurochemical aftermath of amateur boxing. Arch Neurol 2006, 63(9):1277-1280.

13. Zetterberg H, Pedersen M, Lind K, Svensson M, Rolstad S, Eckerstrom C, Syversen S, Mattsson UB, Ysander C, Mattsson N et al: Intra-individual stability of CSF biomarkers for Alzheimer's disease over two years. J Alzheimers Dis 2007, 12(3):255260.

14. Hampel H, Buerger K, Zinkowski R, Teipel SJ, Goernitz A, Andreasen N, Sjoegren M, DeBernardis J, Kerkman D, Ishiguro K et al: Measurement of phosphorylated tau epitopes in the differential diagnosis of Alzheimer disease: a comparative cerebrospinal fluid study. Arch Gen Psychiatry 2004, 61(1):95-102.

15. Mattsson N, Savman K, Osterlundh G, Blennow K, Zetterberg H: Converging molecular pathways in human neural development and degeneration. Neurosci Res 2009.

16. Grahn A, Hagberg L, Nilsson S, Blennow K, Zetterberg H, Studahl M: Cerebrospinal fluid biomarkers in patients with varicella-zoster virus CNS infections. J Neurol 2013, 260(7):1813-1821.

17. Kondziella D, Zetterberg H: Hyperphosphorylation of tau protein in superficial CNS siderosis. J Neurol Sci 2008, 273(1-2):130-132.

18. Ikeda T, Noto D, Noguchi-Shinohara M, Ono K, Takahashi K, Ishida C, Yoshita M, Kawaguchi M, Kawahara N, Iwasa K et al: CSF tau protein is a useful marker for effective treatment of superficial siderosis of the central nervous system: two case reports. Clin Neurol Neurosurg 2010, 112(1):62-64.

19. Buerger K, Ewers M, Pirttila T, Zinkowski R, Alafuzoff I, Teipel SJ, DeBernardis J, Kerkman D, McCulloch C, Soininen $\mathrm{H}$ et al: CSF phosphorylated tau protein correlates with neocortical neurofibrillary pathology in Alzheimer's disease. Brain 2006, 129(Pt 11):3035-3041. 
20. Seppala TT, Nerg O, Koivisto AM, Rummukainen J, Puli L, Zetterberg H, Pyykko OT, Helisalmi S, Alafuzoff I, Hiltunen M et al: CSF biomarkers for Alzheimer disease correlate with cortical brain biopsy findings. Neurology 2012, 78(20):1568-1575.

21. Wang L, Fagan AM, Shah AR, Beg MF, Csernansky JG, Morris JC, Holtzman DM: Cerebrospinal fluid proteins predict longitudinal hippocampal degeneration in earlystage dementia of the Alzheimer type. Alzheimer Dis Assoc Disord 2012, 26(4):314321.

22. Glodzik L, Mosconi L, Tsui W, de Santi S, Zinkowski R, Pirraglia E, Rich KE, McHugh P, Li Y, Williams S et al: Alzheimer's disease markers, hypertension, and gray matter damage in normal elderly. Neurobiol Aging 2012, 33(7):1215-1227.

23. Trojanowski JQ, Schuck T, Schmidt ML, Lee VM: Distribution of tau proteins in the normal human central and peripheral nervous system. J Histochem Cytochem 1989, 37(2):209-215.

24. Smith R, Wibom M, Olsson T, Hagerstrom D, Jogi J, Rabinovici GD, Hansson O: Posterior Accumulation of Tau and Concordant Hypometabolism in an Early-Onset Alzheimer's Disease Patient with Presenilin-1 Mutation. J Alzheimers Dis 2016, 51(2):339-343.

25. Romero I, Jorgensen P, Bolwig G, Fraser PE, Rogaeva E, Mann D, Havsager AM, Jorgensen AL: A presenilin-1 Thr116Asn substitution in a family with early-onset Alzheimer's disease. Neuroreport 1999, 10(11):2255-2260.

26. Blom ES, Giedraitis V, Zetterberg H, Fukumoto H, Blennow K, Hyman BT, Irizarry MC, Wahlund LO, Lannfelt L, Ingelsson M: Rapid progression from mild cognitive impairment to Alzheimer's disease in subjects with elevated levels of tau in cerebrospinal fluid and the APOE epsilon4/epsilon4 genotype. Dement Geriatr Cogn Disord 2009, 27(5):458-464.

27. Wallin AK, Blennow K, Zetterberg H, Londos E, Minthon L, Hansson O: CSF biomarkers predict a more malignant outcome in Alzheimer disease. Neurology 2010, 74(19):1531-1537.

28. Skillback T, Rosen C, Asztely F, Mattsson N, Blennow K, Zetterberg H: Diagnostic performance of cerebrospinal fluid total tau and phosphorylated tau in CreutzfeldtJakob disease: results from the Swedish Mortality Registry. JAMA Neurol 2014, 71(4):476-483.

29. Randall J, Mortberg E, Provuncher GK, Fournier DR, Duffy DC, Rubertsson S, Blennow K, Zetterberg H, Wilson DH: Tau proteins in serum predict neurological outcome after hypoxic brain injury from cardiac arrest: results of a pilot study. Resuscitation 2013, 84(3):351-356.

30. Zetterberg H, Wilson D, Andreasson U, Minthon L, Blennow K, Randall J, Hansson O: Plasma tau levels in Alzheimer's disease. Alzheimers Res Ther 2013, 5(2):9.

31. Shahim P, Tegner Y, Wilson DH, Randall J, Skillback T, Pazooki D, Kallberg B, Blennow K, Zetterberg H: Blood biomarkers for brain injury in concussed professional ice hockey players. JAMA Neurol 2014, 71(6):684-692.

32. Bogoslovsky T, Wilson D, Chen Y, Hanlon D, Gill J, Jeromin A, Song L, Moore C, Gong Y, Kenney K et al: Increases of Plasma Levels of Glial Fibrillary Acidic Protein, Tau, and Amyloid beta up to 90 Days after Traumatic Brain Injury. J Neurotrauma 2016.

33. Olivera A, Lejbman N, Jeromin A, French LM, Kim HS, Cashion A, Mysliwiec V, Diaz-Arrastia R, Gill J: Peripheral Total Tau in Military Personnel Who Sustain Traumatic Brain Injuries During Deployment. JAMA Neurol 2015, 72(10):1109-1116.

34. Arendt T, Stieler J, Holzer M: Tau and Tauopathies. Brain Res Bull 2016. 
35. Meredith JE, Jr., Sankaranarayanan S, Guss V, Lanzetti AJ, Berisha F, Neely RJ, Slemmon JR, Portelius E, Zetterberg H, Blennow K et al: Characterization of Novel CSF Tau and ptau Biomarkers for Alzheimer's Disease. PLoS ONE 2013, 8(10):e76523.

36. Luk C, Compta Y, Magdalinou N, Marti MJ, Hondhamuni G, Zetterberg H, Blennow K, Constantinescu R, Pijnenburg Y, Mollenhauer B et al: Development and assessment of sensitive immuno-PCR assays for the quantification of cerebrospinal fluid three- and four-repeat tau isoforms in tauopathies. J Neurochem 2012 , 123(3):396-405.

37. Lewczuk P, Lelental N, Lachmann I, Holzer M, Flach K, Brandner S, Engelborghs S, Teunissen CE, Zetterberg H, Molinuevo JL et al: Non-Phosphorylated Tau as a Potential Biomarker of Alzheimer's Disease: Analytical and Diagnostic Characterization. J Alzheimers Dis 2016.

38. Russell CL, Mitra V, Hansson K, Blennow K, Gobom J, Zetterberg H, Hiltunen M, Ward M, Pike I: Comprehensive Quantitative Profiling of Tau and Phosphorylated Tau Peptides in Cerebrospinal Fluid by Mass Spectrometry Provides New Biomarker Candidates. J Alzheimers Dis 2016.

39. Barthelemy NR, Gabelle A, Hirtz C, Fenaille F, Sergeant N, Schraen-Maschke S, Vialaret J, Buee L, Junot C, Becher F et al: Differential Mass Spectrometry Profiles of Tau Protein in the Cerebrospinal Fluid of Patients with Alzheimer's Disease, Progressive Supranuclear Palsy, and Dementia with Lewy Bodies. J Alzheimers Dis 2016, 51(4):1033-1043.

40. McAvoy T, Lassman ME, Spellman DS, Ke Z, Howell BJ, Wong O, Zhu L, Tanen M, Struyk A, Laterza OF: Quantification of tau in cerebrospinal fluid by immunoaffinity enrichment and tandem mass spectrometry. Clin Chem 2014, 60(4):683-689. 
Table 1. CSF total- and phospho-tau concentrations in neurodegenerative diseases and common differential diagnoses

\begin{tabular}{|l|l|l|}
\hline Disease & Total-tau concentration & Phospho-tau concentration \\
\hline Alzheimer's disease & Medium to strong increase & Medium to strong increase \\
\hline Vascular dementia & Normal to strong increase & Normal \\
\hline Frontotemporal dementia & Normal to slight increase & Normal \\
\hline Dementia with Lewy bodies & Normal to slight increase & Normal \\
\hline Parkinson's disease & Normal & Normal \\
\hline $\begin{array}{l}\text { Atypical parkinsonian } \\
\text { disorders }\end{array}$ & Normal to slight increase & Normal \\
\hline Creutzfeldt-Jakob's disease & Very strong increase & Normal \\
\hline $\begin{array}{l}\text { Stable mild cognitive } \\
\text { impairment }\end{array}$ & Normal & Normal \\
\hline Normal aging & & Normal \\
\hline Depression & Normal & Normal \\
\hline
\end{tabular}

